\title{
Search for Large Extra Dimensions via Single Photon plus Missing Energy Final States at $\sqrt{s}=1.96 \mathrm{TeV}$
}

V. M. Abazov,${ }^{36}$ B. Abbott, ${ }^{75}$ M. Abolins, ${ }^{65}$ B. S. Acharya,${ }^{29}$ M. Adams,${ }^{51}$ T. Adams,${ }^{49}$ E. Aguilo, ${ }^{6}$ S. H. Ahn, ${ }^{31}$ M. Ahsan, ${ }^{59}$ G. D. Alexeev, ${ }^{36}$ G. Alkhazov, ${ }^{40}$ A. Alton, ${ }^{64, *}$ G. Alverson, ${ }^{63}$ G. A. Alves, ${ }^{2}$ M. Anastasoaie,${ }^{35}$ L. S. Ancu, ${ }^{35}$ T. Andeen ${ }^{53}$ S. Anderson,${ }^{45}$ B. Andrieu,${ }^{17}$ M. S. Anzelc, ${ }^{53}$ M. Aoki,${ }^{50}$ Y. Arnoud, ${ }^{14}$ M. Arov,${ }^{60}$ M. Arthaud, ${ }^{18}$ A. Askew, ${ }^{49}$ B. Ásman, ${ }^{41}$ A. C. S. Assis Jesus, ${ }^{3}$ O. Atramentov, ${ }^{49}$ C. Avila,${ }^{8}$ C. Ay, ${ }^{24}$ F. Badaud, ${ }^{13}$ A. Baden, ${ }^{61}$ L. Bagby, ${ }^{50}$ B. Baldin,${ }^{50}$ D. V. Bandurin ${ }^{59}$ P. Banerjee ${ }^{29}$ S. Banerjee ${ }^{29}$ E. Barberis, ${ }^{63}$ A.-F. Barfuss, ${ }^{15}$ P. Bargassa,${ }^{80}$ P. Baringer, ${ }^{58}$ J. Barreto, ${ }^{2}$

J.F. Bartlett, ${ }^{50}$ U. Bassler, ${ }^{18}$ D. Bauer,${ }^{43}$ S. Beale, ${ }^{6}$ A. Bean,${ }^{58} \mathrm{M}$. Begalli, ${ }^{3} \mathrm{M}$. Begel, ${ }^{73} \mathrm{C}$. Belanger-Champagne, ${ }^{41}$ L. Bellantoni, ${ }^{50}$ A. Bellavance, ${ }^{50}$ J. A. Benitez ${ }^{65}$ S. B. Beri, ${ }^{27}$ G. Bernardi, ${ }^{17}$ R. Bernhard,${ }^{23}$ I. Bertram,${ }^{42}$ M. Besançon, ${ }^{18}$ R. Beuselinck, ${ }^{43}$ V. A. Bezzubov, ${ }^{39}$ P. C. Bhat,${ }^{50}$ V. Bhatnagar, ${ }^{27}$ C. Biscarat,${ }^{20}$ G. Blazey, ${ }^{52}$ F. Blekman, ${ }^{43}$ S. Blessing,${ }^{49}$ D. Bloch,${ }^{19}$ K. Bloom,${ }^{67}$ A. Boehnlein, ${ }^{50}$ D. Boline, ${ }^{62}$ T. A. Bolton, ${ }^{59}$ G. Borissov, ${ }^{42}$ T. Bose, ${ }^{77}$ A. Brandt,${ }^{78}$ R. Brock, ${ }^{65}$ G. Brooijmans ${ }^{70}$ A. Bross ${ }^{50}$ D. Brown, ${ }^{81}$ N. J. Buchanan, ${ }^{49}$ D. Buchholz,${ }^{53}$ M. Buehler, ${ }^{81}$ V. Buescher, ${ }^{22}$ V. Bunichev,${ }^{38}$ S. Burdin, ${ }^{42, \dagger}$ S. Burke, ${ }^{45}$ T. H. Burnett, ${ }^{82}$ C. P. Buszello, ${ }^{43}$ J. M. Butler, ${ }^{62}$ P. Calfayan, ${ }^{25}$ S. Calvet, ${ }^{16}$ J. Cammin, ${ }^{71}$ E. Carrera, ${ }^{49}$ W. Carvalho, ${ }^{3}$ B. C. K. Casey,${ }^{50}$ H. Castilla-Valdez,${ }^{33}$ S. Chakrabarti, ${ }^{18}$ D. Chakraborty, ${ }^{52}$ K. Chan, ${ }^{6}$ K. M. Chan ${ }^{55}$ A. Chandra, ${ }^{48}$ F. Charles, ${ }^{19}, * *$ E. Cheu, ${ }^{45}$ F. Chevallier, ${ }^{14}$ D. K. Cho, ${ }^{62}$ S. Choi,${ }^{32}$ B. Choudhary, ${ }^{28}$ L. Christofek, ${ }^{77}$ T. Christoudias, ${ }^{43}$ S. Cihangir,${ }^{50}$ D. Claes,${ }^{67}$ Y. Coadou, ${ }^{6}$ M. Cooke, ${ }^{80}$ W. E. Cooper, ${ }^{50}$ M. Corcoran, ${ }^{80}$ F. Couderc, ${ }^{18}$ M.-C. Cousinou, ${ }^{15}$ S. Crépé-Renaudin, ${ }^{14}$ D. Cutts, ${ }^{77}$ M. Ćwiok, ${ }^{30}$ H. da Motta, ${ }^{2}$ A. Das,${ }^{45}$ G. Davies, ${ }^{43}$ K. De,${ }^{78}$ S. J. de Jong, ${ }^{35}$ E. De La Cruz-Burelo,${ }^{64}$ C. De Oliveira Martins, ${ }^{3}$ J. D. Degenhardt, ${ }^{64}$ F. Déliot, ${ }^{18}$ M. Demarteau,${ }^{50}$ R. Demina, ${ }^{71}$ D. Denisov, ${ }^{50}$ S. P. Denisov, ${ }^{39}$ S. Desai,${ }^{50}$ H. T. Diehl,${ }^{50}$ M. Diesburg, ${ }^{50}$ A. Dominguez, ${ }^{67}$ H. Dong, ${ }^{72}$ L. V. Dudko, ${ }^{38}$ L. Duflot, ${ }^{16}$ S. R. Dugad ${ }^{29}$ D. Duggan ${ }^{49}$ A. Duperrin,,${ }^{15}$ J. Dyer ${ }^{65}$ A. Dyshkant, ${ }^{52}$ M. Eads,${ }^{67}$ D. Edmunds, ${ }^{65}$ J. Ellison, ${ }^{48}$ V. D. Elvira,${ }^{50}$ Y. Enari,${ }^{77}$ S. Eno,${ }^{61}$ P. Ermolov,${ }^{38}$ H. Evans,${ }^{54}$ A. Evdokimov, ${ }^{73}$ V. N. Evdokimov, ${ }^{39}$ A. V. Ferapontov, ${ }^{59}$ T. Ferbel, ${ }^{71}$ F. Fiedler, ${ }^{24}$ F. Filthaut, ${ }^{35}$ W. Fisher, ${ }^{50}$ H. E. Fisk,${ }^{50}$ M. Fortner, ${ }^{52}$ H. Fox,${ }^{42}$ S. Fu, ${ }^{50}$ S. Fuess,${ }^{50}$ T. Gadfort,${ }^{70}$ C. F. Galea,${ }^{35}$ E. Gallas, ${ }^{50}$ C. Garcia, ${ }^{71}$ A. Garcia-Bellido, ${ }^{82}$ V. Gavrilov, ${ }^{37}$ P. Gay, ${ }^{13}$ W. Geist, ${ }^{19}$ D. Gelé, ${ }^{19}$ C. E. Gerber ${ }^{51}$ Y. Gershtein, ${ }^{49}$ D. Gillberg, ${ }^{6}$ G. Ginther, ${ }^{71}$ N. Gollub, ${ }^{41}$ B. Gómez, ${ }^{8}$

A. Goussiou, ${ }^{82}$ P. D. Grannis, ${ }^{72}$ H. Greenlee, ${ }^{50}$ Z. D. Greenwood,${ }^{60}$ E. M. Gregores ${ }^{4}$ G. Grenier,${ }^{20}$ Ph. Gris, ${ }^{13}$ J.-F. Grivaz, ${ }^{16}$ A. Grohsjean, ${ }^{25}$ S. Grünendahl,${ }^{50}$ M. W. Grünewald, ${ }^{30}$ F. Guo, ${ }^{72}$ J. Guo,${ }^{72}$ G. Gutierrez,${ }^{50}$ P. Gutierrez,${ }^{75}$ A. Haas, ${ }^{70}$ N. J. Hadley, ${ }^{61}$ P. Haefner, ${ }^{25}$ S. Hagopian, ${ }^{49}$ J. Haley ${ }^{68}$ I. Hall, ${ }^{65}$ R. E. Hall, ${ }^{47}$ L. Han,${ }^{7}$ K. Harder, ${ }^{44}$ A. Harel, ${ }^{71}$ R. Harrington, ${ }^{63}$ J. M. Hauptman, ${ }^{57}$ R. Hauser, ${ }^{65}$ J. Hays, ${ }^{43}$ T. Hebbeker, ${ }^{21}$ D. Hedin, ${ }^{52}$ J. G. Hegeman, ${ }^{34}$

J. M. Heinmiller, ${ }^{51}$ A. P. Heinson, ${ }^{48}$ U. Heintz,${ }^{62}$ C. Hensel, ${ }^{58}$ K. Herner, ${ }^{72}$ G. Hesketh, ${ }^{63}$ M. D. Hildreth, ${ }^{55}$ R. Hirosky, ${ }^{81}$ J. D. Hobbs, ${ }^{72}$ B. Hoeneisen, ${ }^{12}$ H. Hoeth, ${ }^{26}$ M. Hohlfeld, ${ }^{22}$ S. J. Hong, ${ }^{31}$ S. Hossain, ${ }^{75}$ P. Houben, ${ }^{34}$ Y. Hu, ${ }^{72}$ Z. Hubacek, ${ }^{10}$ V. Hynek, ${ }^{9}$ I. Iashvili, ${ }^{69}$ R. Illingworth ${ }^{50}$ A. S. Ito, ${ }^{50}$ S. Jabeen, ${ }^{62}$ M. Jaffré, ${ }^{16}$ S. Jain, ${ }^{75}$ K. Jakobs,${ }^{23}$ C. Jarvis,${ }^{61}$ R. Jesik, ${ }^{43}$ K. Johns,${ }^{45}$ C. Johnson, ${ }^{70}$ M. Johnson, ${ }^{50}$ A. Jonckheere, ${ }^{50}$ P. Jonsson, ${ }^{43}$ A. Juste, ${ }^{50}$ E. Kajfasz, ${ }^{15}$ A. M. Kalinin, ${ }^{36}$ J. M. Kalk, ${ }^{60}$ S. Kappler, ${ }^{21}$ D. Karmanov, ${ }^{38}$ P. A. Kasper ${ }^{50}$ I. Katsanos, ${ }^{70}$ D. Kau,${ }^{49}$ V. Kaushik, ${ }^{78}$ R. Kehoe, ${ }^{79}$ S. Kermiche, ${ }^{15}$ N. Khalatyan, ${ }^{50}$ A. Khanov, ${ }^{76}$ A. Kharchilava, ${ }^{69}$ Y. M. Kharzheev, ${ }^{36}$ D. Khatidze, ${ }^{70}$ T. J. Kim, ${ }^{31}$ M. H. Kirby, ${ }^{53}$ M. Kirsch ${ }^{21}$ B. Klima,${ }^{50}$ J. M. Kohli, ${ }^{27}$ J.-P. Konrath, ${ }^{23}$ V. M. Korablev, ${ }^{39}$ A. V. Kozelov, ${ }^{39}$ J. Kraus, ${ }^{65}$ D. Krop, ${ }^{54}$ T. Kuhl,${ }^{24}$ A. Kumar, ${ }^{69}$ A. Kupco, ${ }^{11}$ T. Kurča, ${ }^{20}$ J. Kvita, ${ }^{9}$ F. Lacroix, ${ }^{13}$ D. Lam, ${ }^{55}$ S. Lammers, ${ }^{70}$ G. Landsberg, ${ }^{77}$ P. Lebrun, ${ }^{20}$ W. M. Lee, ${ }^{50}$ A. Leflat, ${ }^{38}$ J. Lellouch,,${ }^{17}$ J. Leveque,${ }^{45}$ J. Li,${ }^{78}$ L. Li ${ }^{48}$ Q. Z. Li,${ }^{50}$ S. M. Lietti, J. G. R. Lima,${ }^{52}$ D. Lincoln, ${ }^{50}$ J. Linnemann, ${ }^{65}$ V. V. Lipaev, ${ }^{39}$ R. Lipton, ${ }^{50}$ Y. Liu, ${ }^{7}$ Z. Liu, ${ }^{6}$ A. Lobodenko, ${ }^{40}$

M. Lokajicek, ${ }^{11}$ P. Love, ${ }^{42}$ H. J. Lubatti, ${ }^{82}$ R. Luna, ${ }^{3}$ A. L. Lyon, ${ }^{50}$ A. K. A. Maciel, ${ }^{2}$ D. Mackin, ${ }^{80}$ R. J. Madaras, ${ }^{46}$ P. Mättig, ${ }^{26}$ C. Magass, ${ }^{21}$ A. Magerkurth, ${ }^{64}$ P. K. Mal, ${ }^{82}$ H. B. Malbouisson, ${ }^{3}$ S. Malik,${ }^{67}$ V. L. Malyshev, ${ }^{36}$ H. S. Mao, ${ }^{50}$ Y. Maravin, ${ }^{59}$ B. Martin, ${ }^{14}$ R. McCarthy, ${ }^{72}$ A. Melnitchouk, ${ }^{66}$ L. Mendoza, ${ }^{8}$ P. G. Mercadante, ${ }^{5}$ M. Merkin, ${ }^{38}$

K. W. Merritt, ${ }^{50}$ A. Meyer, ${ }^{21}$ J. Meyer, ${ }^{22,8}$ T. Millet, ${ }^{20}$ J. Mitrevski, ${ }^{70}$ J. Molina, ${ }^{3}$ R. K. Mommsen, ${ }^{44}$ N. K. Mondal, ${ }^{29}$

R.W. Moore, ${ }^{6}$ T. Moulik, ${ }^{58}$ G. S. Muanza, ${ }^{20}$ M. Mulders,${ }^{50}$ M. Mulhearn,${ }^{70}$ O. Mundal, ${ }^{22}$ L. Mundim, ${ }^{3}$ E. Nagy, ${ }^{15}$ M. Naimuddin,${ }^{50}$ M. Narain, ${ }^{77}$ N. A. Naumann, ${ }^{35}$ H. A. Neal,${ }^{64}$ J. P. Negret, ${ }^{8}$ P. Neustroev, ${ }^{40}$ H. Nilsen, ${ }^{23}$ H. Nogima, ${ }^{3}$ S. F. Novaes, ${ }^{5}$ T. Nunnemann, ${ }^{25}$ V. O'Dell, ${ }^{50}$ D. C. O'Neil, ${ }^{6}$ G. Obrant,${ }^{40}$ C. Ochando, ${ }^{16}$ D. Onoprienko, ${ }^{59}$ N. Oshima, ${ }^{50}$ N. Osman, ${ }^{43}$ J. Osta, ${ }^{55}$ R. Otec, ${ }^{10}$ G. J. Otero y Garzón, ${ }^{50}$ M. Owen, ${ }^{44}$ P. Padley ${ }^{80}$ M. Pangilinan, ${ }^{77}$ N. Parashar, ${ }^{56}$ S.-J. Park ${ }^{71}$ S. K. Park,${ }^{31}$ J. Parsons,${ }^{70}$ R. Partridge,${ }^{77}$ N. Parua,${ }^{54}$ A. Patwa ${ }^{73}$ G. Pawloski ${ }^{80}$ B. Penning,${ }^{23}$ M. Perfilov,${ }^{38}$ K. Peters, ${ }^{44}$ Y. Peters, ${ }^{26}$ P. Pétroff, ${ }^{16}$ M. Petteni, ${ }^{43}$ R. Piegaia, ${ }^{1}$ J. Piper, ${ }^{65}$ M.-A. Pleier, ${ }^{22}$ P. L. M. Podesta-Lerma, ${ }^{33, *}$ V. M. Podstavkov, ${ }^{50}$ Y. Pogorelov, ${ }^{55}$ M.-E. Pol, ${ }^{2}$ P. Polozov,${ }^{37}$ B. G. Pope,${ }^{65}$ A. V. Popov, ${ }^{39}$ C. Potter,${ }^{6}$ 
W. L. Prado da Silva, ${ }^{3}$ H. B. Prosper, ${ }^{49}$ S. Protopopescu, ${ }^{73}$ J. Qian, ${ }^{64}$ A. Quadt, ${ }^{22, \S}$ B. Quinn, ${ }^{66}$ A. Rakitine, ${ }^{42}$ M. S. Rangel, ${ }^{2}$ K. Ranjan, ${ }^{28}$ P. N. Ratoff, ${ }^{42}$ P. Renkel, ${ }^{79}$ S. Reucroft, ${ }^{63}$ P. Rich, ${ }^{44}$ J. Rieger, ${ }^{54}$ M. Rijssenbeek, ${ }^{72}$ I. Ripp-Baudot, ${ }^{19}$ F. Rizatdinova, ${ }^{76}$ S. Robinson, ${ }^{43}$ R. F. Rodrigues, ${ }^{3}$ M. Rominsky, ${ }^{75}$ C. Royon, ${ }^{18}$ P. Rubinov, ${ }^{50}$ R. Ruchti, ${ }^{55}$ G. Safronov, ${ }^{37}$ G. Sajot, ${ }^{14}$ A. Sánchez-Hernández, ${ }^{33}$ M. P. Sanders, ${ }^{17}$ A. Santoro, ${ }^{3}$ G. Savage, ${ }^{50}$ L. Sawyer, ${ }^{60}$ T. Scanlon, ${ }^{43}$ D. Schaile, ${ }^{25}$ R. D. Schamberger, ${ }^{72}$ Y. Scheglov, ${ }^{40}$ H. Schellman, ${ }^{53}$ T. Schliephake, ${ }^{26}$ C. Schwanenberger, ${ }^{44}$ A. Schwartzman, ${ }^{68}$ R. Schwienhorst, ${ }^{65}$ J. Sekaric, ${ }^{49}$ H. Severini, ${ }^{75}$ E. Shabalina, ${ }^{51}$ M. Shamim, ${ }^{59}$ V. Shary, ${ }^{18}$ A. A. Shchukin, ${ }^{39}$ R. K. Shivpuri, ${ }^{28}$ V. Siccardi, ${ }^{19}$ V. Simak, ${ }^{10}$ V. Sirotenko, ${ }^{50}$ P. Skubic, ${ }^{75}$ P. Slattery, ${ }^{71}$ D. Smirnov, ${ }^{55}$ G. R. Snow, ${ }^{67}$ J. Snow, ${ }^{74}$ S. Snyder, ${ }^{73}$ S. Söldner-Rembold, ${ }^{44}$ L. Sonnenschein, ${ }^{17}$ A. Sopczak, ${ }^{42}$ M. Sosebee, ${ }^{78}$ K. Soustruznik, ${ }^{9}$ B. Spurlock, ${ }^{78}$ J. Stark, ${ }^{14}$ J. Steele, ${ }^{60}$ V. Stolin, ${ }^{37}$ D. A. Stoyanova, ${ }^{39}$ J. Strandberg, ${ }^{64}$ S. Strandberg, ${ }^{41}$ M. A. Strang, ${ }^{69}$ E. Strauss, ${ }^{72}$ M. Strauss, ${ }^{75}$ R. Ströhmer, ${ }^{25}$ D. Strom, ${ }^{53}$ L. Stutte, ${ }^{50}$ S. Sumowidagdo, ${ }^{49}$ P. Svoisky, ${ }^{55}$ A. Sznajder, ${ }^{3}$ P. Tamburello, ${ }^{45}$ A. Tanasijczuk, ${ }^{1}$ W. Taylor, ${ }^{6}$ J. Temple, ${ }^{45}$ B. Tiller, ${ }^{25}$ F. Tissandier, ${ }^{13}$ M. Titov, ${ }^{18}$ V. V. Tokmenin, ${ }^{36}$ T. Toole, ${ }^{61}$ I. Torchiani, ${ }^{23}$ T. Trefzger, ${ }^{24}$ D. Tsybychev, ${ }^{72}$ B. Tuchming, ${ }^{18}$ C. Tully, ${ }^{68}$ P. M. Tuts, ${ }^{70}$

R. Unalan, ${ }^{65}$ L. Uvarov, ${ }^{40}$ S. Uvarov, ${ }^{40}$ S. Uzunyan, ${ }^{52}$ B. Vachon, ${ }^{6}$ P. J. van den Berg, ${ }^{34}$ R. Van Kooten, ${ }^{54}$ W. M. van Leeuwen, ${ }^{34}$ N. Varelas, ${ }^{51}$ E. W. Varnes, ${ }^{45}$ I. A. Vasilyev, ${ }^{39}$ M. Vaupel, ${ }^{26}$ P. Verdier, ${ }^{20}$ L. S. Vertogradov, ${ }^{36}$ M. Verzocchi, ${ }^{50}$ F. Villeneuve-Seguier, ${ }^{43}$ P. Vint, ${ }^{43}$ P. Vokac, ${ }^{10}$ E. Von Toerne, ${ }^{59}$ M. Voutilainen, ${ }^{68, \|}$ R. Wagner, ${ }^{68}$ H. D. Wahl, ${ }^{49}$ L. Wang, ${ }^{61}$ M. H. L. S. Wang, ${ }^{50}$ J. Warchol, ${ }^{55}$ G. Watts, ${ }^{82}$ M. Wayne, ${ }^{55}$ G. Weber, ${ }^{24}$ M. Weber, ${ }^{50}$ L. Welty-Rieger, ${ }^{54}$ A. Wenger, ${ }^{23,4 l}$ N. Wermes, ${ }^{22}$ M. Wetstein, ${ }^{61}$ A. White, ${ }^{78}$ D. Wicke, ${ }^{26}$ G. W. Wilson, ${ }^{58}$ S. J. Wimpenny, ${ }^{48}$ M. Wobisch, ${ }^{60}$ D. R. Wood, ${ }^{63}$ T. R. Wyatt, ${ }^{44}$ Y. Xie, ${ }^{77}$ S. Yacoob, ${ }^{53}$ R. Yamada, ${ }^{50}$ M. Yan, ${ }^{61}$ T. Yasuda, ${ }^{50}$ Y. A. Yatsunenko, ${ }^{36}$ K. Yip, ${ }^{73}$ H. D. Yoo, ${ }^{77}$ S. W. Youn, ${ }^{53}$ J. Yu, ${ }^{78}$ A. Zatserklyaniy, ${ }^{52}$ C. Zeitnitz, ${ }^{26}$ T. Zhao, ${ }^{82}$ B. Zhou, ${ }^{64}$ J. Zhu, ${ }^{72}$ M. Zielinski, ${ }^{71}$ D. Zieminska, ${ }^{54}$ A. Zieminski, ${ }^{54 * *}$ L. Zivkovic, $^{70}$ V. Zutshi, ${ }^{52}$ and E. G. Zverev ${ }^{38}$

\title{
(D0 Collaboration)
}

\author{
${ }^{1}$ Universidad de Buenos Aires, Buenos Aires, Argentina \\ ${ }^{2}$ LAFEX, Centro Brasileiro de Pesquisas Físicas, Rio de Janeiro, Brazil \\ ${ }^{3}$ Universidade do Estado do Rio de Janeiro, Rio de Janeiro, Brazil \\ ${ }^{4}$ Universidade Federal do ABC, Santo André, Brazil \\ ${ }^{5}$ Instituto de Física Teórica, Universidade Estadual Paulista, São Paulo, Brazil \\ ${ }^{6}$ University of Alberta, Edmonton, Alberta, Canada, Simon Fraser University, Burnaby, British Columbia, Canada, \\ York University, Toronto, Ontario, Canada, \\ and McGill University, Montreal, Quebec, Canada \\ ${ }^{7}$ University of Science and Technology of China, Hefei, People's Republic of China \\ ${ }^{8}$ Universidad de los Andes, Bogotá, Colombia \\ ${ }^{9}$ Center for Particle Physics, Charles University, Prague, Czech Republic \\ ${ }^{10}$ Czech Technical University, Prague, Czech Republic \\ ${ }^{11}$ Center for Particle Physics, Institute of Physics, Academy of Sciences of the Czech Republic, Prague, Czech Republic \\ ${ }^{12}$ Universidad San Francisco de Quito, Quito, Ecuador \\ ${ }^{13}$ LPC, Univ Blaise Pascal, CNRS/IN2P3, Clermont, France \\ ${ }^{14}$ LPSC, Université Joseph Fourier Grenoble 1, CNRS/IN2P3, Institut National Polytechnique de Grenoble, France \\ ${ }^{15}$ CPPM, IN2P3/CNRS, Université de la Méditerranée, Marseille, France \\ ${ }^{16}$ LAL, Univ Paris-Sud, IN2P3/CNRS, Orsay, France \\ ${ }^{17}$ LPNHE, IN2P3/CNRS, Universités Paris VI and VII, Paris, France \\ ${ }^{18}$ DAPNIA/Service de Physique des Particules, CEA, Saclay, France \\ ${ }^{19}$ IPHC, Université Louis Pasteur et Université de Haute Alsace, CNRS/IN2P3, Strasbourg, France \\ ${ }^{20} I P N L$, Université Lyon 1, CNRS/IN2P3, Villeurbanne, France and Université de Lyon, Lyon, France \\ ${ }^{21}$ III. Physikalisches Institut A, RWTH Aachen, Aachen, Germany \\ ${ }^{22}$ Physikalisches Institut, Universität Bonn, Bonn, Germany \\ ${ }^{23}$ Physikalisches Institut, Universität Freiburg, Freiburg, Germany \\ ${ }^{24}$ Institut für Physik, Universität Mainz, Mainz, Germany \\ ${ }^{25}$ Ludwig-Maximilians-Universität München, München, Germany \\ ${ }^{26}$ Fachbereich Physik, University of Wuppertal, Wuppertal, Germany \\ ${ }^{27}$ Panjab University, Chandigarh, India \\ ${ }^{28}$ Delhi University, Delhi, India \\ ${ }^{29}$ Tata Institute of Fundamental Research, Mumbai, India \\ ${ }^{30}$ University College Dublin, Dublin, Ireland \\ ${ }^{31}$ Korea Detector Laboratory, Korea University, Seoul, Korea
}




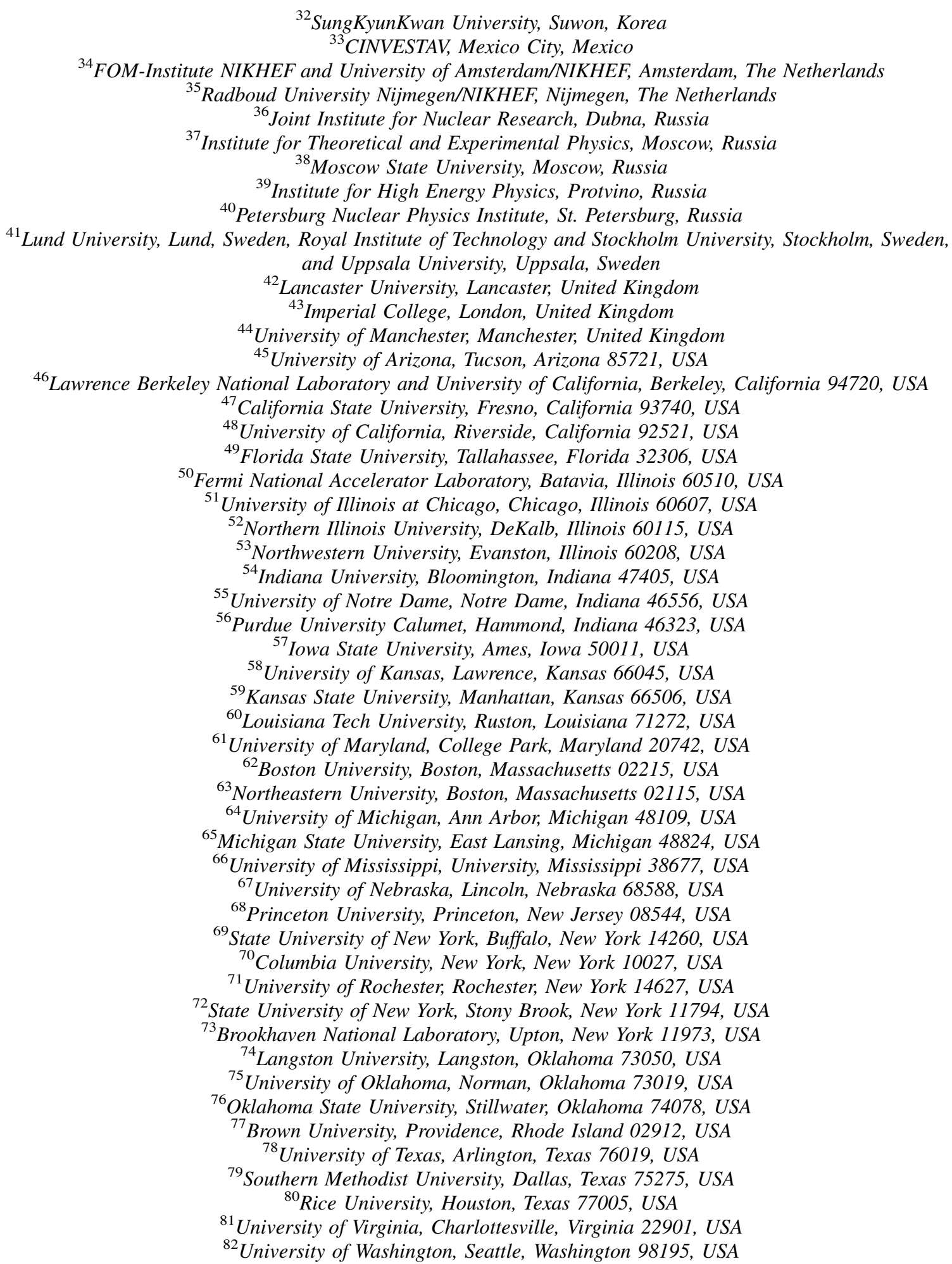

We report on a search for large extra dimensions in a data sample of approximately $1 \mathrm{fb}^{-1}$ of $p \bar{p}$ collisions at $\sqrt{s}=1.96 \mathrm{TeV}$. We investigate Kaluza-Klein graviton production with a photon and missing transverse energy in the final state. At the $95 \%$ C.L. we set limits on the fundamental mass scale $M_{D}$ from 884 to $778 \mathrm{GeV}$ for two to eight extra dimensions. 
Arkani-Hamed, Dimopoulos, and Dvali (ADD) [1] made the first attempt to solve the hierarchy problem of the standard model (SM) by postulating the existence of $n$ new large extra spatial dimensions (LED). In this approach, the SM particles are confined to a 3-dimensional brane while gravity is diluted in the larger volume. The size of the compactified extra space $(R)$, the effective Planck scale in the 4-dimensional space-time $\left(M_{\mathrm{Pl}}\right)$, and the fundamental Planck scale in the $(4+n)$-dimensional space-time $\left(M_{D}\right)$, are related by the expression $M_{\mathrm{Pl}}^{2}=$ $8 \pi M_{D}^{n+2} R^{n}$. Because of the compactification of the extra space, the gravitational field appears as a series of quantized energy states, which are referred to as Kaluza-Klein modes. A Kaluza-Klein graviton $\left(G_{K K}\right)$ behaves like a massive, noninteracting, stable particle whose direct production gives an imbalance in the final state momentum as its collider signature.

In this Letter we report the results of a search for LED in the final state with a single photon plus missing transverse energy $\left(\gamma+\not E_{T}\right)$, using data collected with the D0 detector at the Fermilab Tevatron collider. This signature arises from the process $q \bar{q} \rightarrow \gamma G_{K K}$, which is studied in detail in [2]. The CDF collaboration carried out a similar search with $87 \mathrm{pb}^{-1}$ of data, setting $95 \%$ C.L. lower limits on $M_{D}$ of 549,581 , and $601 \mathrm{GeV}$ for 4,6 , and 8 extra dimensions, respectively [3]. Searches for LED in other final states have been performed by collaborations at the Tevatron $[4,5]$ and the CERN LEP collider [6].

The background to the $\gamma+\mathbb{E}_{T}$ signal is dominated by electroweak boson production and noncollision background where muons from the beam halo or cosmic rays undergo bremsstrahlung and produce an energetic photon. The electroweak background is dominated by the processes $Z+\gamma \rightarrow \nu \bar{\nu}+\gamma, W \rightarrow e \nu$ where the electron is misidentified as a photon, $W+\gamma$ where the lepton from the $W$ boson decay is not detected, and $W / Z+$ jet production where the jet is misidentified as a photon.

The D0 detector [7] comprises a central-tracking system with a silicon microstrip tracker (SMT) and a central fiber tracker (CFT), both housed within a $2 \mathrm{~T}$ superconducting solenoidal magnet, with designs optimized for tracking and vertexing at $|\eta|<3$ and $|\eta|<2.5$, respectively, where $\eta$ is the pseudorapidity [8] measured with respect to the geometrical center of the detector. The central preshower system (CPS) is located in front of a liquid-argon-uranium calorimeter and consists of three layers of scintillating strips, providing precise measurement of electromagnetic (EM) shower positions. The calorimeter has a central section (CC) covering $|\eta| \leq 1.1$, and two end sections (EC) that extend coverage to $|\eta| \approx 4.2$ [9]. Each part contains an EM section closest to the interaction region followed by fine and coarse hadronic sections. The EM section has four longitudinal layers and transverse segmentation of $0.1 \times 0.1$ in $\eta$ - $\phi$ space (where $\phi$ is the azimuthal angle), with the exception of the third layer, where it is
$0.05 \times 0.05$. Additionally, scintillators between the CC and EC cryostats provide sampling of developing showers for $1.1<|\eta|<1.4$. The outer muon system, covering $|\eta|<2$, consists of a layer of tracking detectors and scintillation trigger counters in front of $1.8 \mathrm{~T}$ iron toroids, followed by two similar layers after the toroids. The data in this analysis were recorded using triggers requiring at least one energy cluster in the EM section of the calorimeter with transverse momentum $p_{T}>20 \mathrm{GeV}$. The triggers are almost $100 \%$ efficient to select signal events. This set of data corresponds to an integrated luminosity of $1.05 \pm$ $0.06 \mathrm{fb}^{-1}[10]$.

We identify a reconstructed calorimeter cluster as a photon when it satisfies the following requirements: (i) at least $90 \%$ of the energy is deposited in the EM section of the calorimeter; (ii) the calorimeter isolation variable $I=$ $\left[E_{\text {tot }}(0.4)-E_{\text {em }}(0.2)\right] / E_{\text {em }}(0.2)$ is less than 0.07 , where $E_{\text {tot }}(0.4)$ denotes the total energy deposited in the calorimeter in a cone of radius $\mathcal{R}=\sqrt{(\overline{\Delta \eta})^{2}+(\overline{\Delta \phi})^{2}}=0.4$, and $E_{\text {em }}(0.2)$ is the EM energy in a cone of radius $\mathcal{R}=$ 0.2 ; (iii) the track isolation variable, defined as the scalar sum of the transverse momenta of all tracks that originate from the interaction vertex in an annulus of $0.05<\mathcal{R}<$ 0.4 around the cluster, is less than $2 \mathrm{GeV}$; (iv) it has $|\eta|<$ 1.1 ; (v) both transverse and longitudinal shower shapes are consistent with those of a photon; (vi) it has neither an associated track in the central tracking system nor a significant density of hits in the SMT and CFT systems consistent with the presence of a track with $p_{T}$ in agreement with its transverse energy; and (vii) there is an energy deposit in the CPS matched to it. Jets are reconstructed using the iterative midpoint cone algorithm [11] with a cone size of 0.5 . The missing transverse energy is computed from calorimeter cells with $|\eta|<4$ and corrected for the EM and jet energy scales.

The photon sample is obtained by selecting events with only one photon with $p_{T}>90 \mathrm{GeV}$, at least one reconstructed interaction vertex consistent with the measured direction of the photon (see below), and $\mathscr{E}_{T}>70 \mathrm{GeV}$. Additionally, in order to avoid large $\mathbb{E}_{T}$ due to mismeasurement of jet energy, we require no jets with $p_{T}>15 \mathrm{GeV}$. The reduction of the signal efficiency due to the jet veto on initial state radiation has been estimated using PYTHIA [12] to be about $9 \%$. The applied $\mathbb{E}_{T}$ requirement guarantees negligible multijet background in the final candidate sample while being almost fully efficient for signal selection.

We reject events with reconstructed muons and with cosmic ray muons identified using the timing of the signal in the muon scintillation counters or by the presence of a characteristic pattern of hits in the muon drift chambers that is aligned with the reconstructed photon. In order to further reject events with leptons that leave a distinguishable signature in the tracker but that are not reconstructed in the other subsystems of the detector, we impose a requirement on the $p_{T}$ of any isolated track not to be 


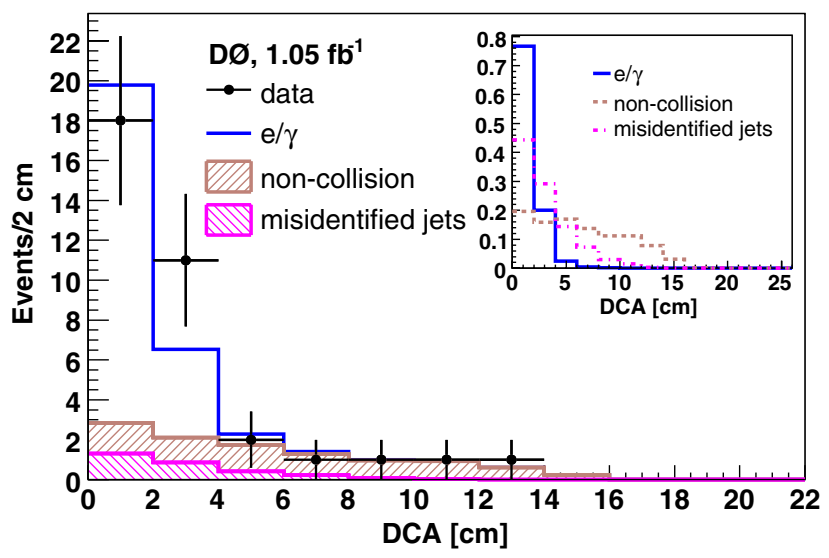

FIG. 1 (color online). DCA distribution for the selected events in data (points with statistical uncertainties). The different histograms represent the estimated background composition from the template fit to this distribution. The inset figure compares the individual template shapes.

greater than $6.5 \mathrm{GeV}$. A track is considered to be isolated if the ratio between the scalar sum of the transverse momenta of all tracks that originate from the interaction vertex in an annulus of $0.1<\mathcal{R}<0.4$ around the track and the $p_{T}$ of the track is less than 0.3 .

The EM pointing algorithm allows calculation of the direction of the EM shower based on the transverse and longitudinal segmentation of the calorimeter and preshower systems. EM pointing is performed independently in the azimuthal and polar planes. The former results in the measurement of the distance of closest approach (DCA) to the $z$ axis (along the beam line), and the latter in the prediction of the $z$ position of the interaction vertex in the event, both with a resolution of about $2 \mathrm{~cm}$. We require that the $z$ coordinate of at least one interaction vertex in the event be within $10 \mathrm{~cm}$ of the position predicted by the pointing algorithm and use the DCA to estimate the remaining background from jet-photon misidentification and noncollision events. Misidentified jets have poor pointing resolution, and therefore a wider DCA distribution compared to electrons or photons. Likewise, one can anticipate the DCA distribution for photon candidates in noncollision events to have an even wider shape. After these requirements, 35 events are selected in the photon sample.

We prepare three DCA distribution templates: the noncollision template, the misidentified jets template, and the $e / \gamma$ template. The first template is obtained from a sample in which a photon candidate, passing the same quality requirements as for the photon sample, is selected from events with no hard scatter (no reconstructed interaction vertex or fewer than three reconstructed tracks), or from events with identified cosmic muons. The misidentified jets template is extracted from the fake photon sample, which fulfills exactly the same requirements as the photon sample except that the photon track isolation requirement is inverted. This sample is dominated by misidentified jets.
TABLE I. Data and estimated backgrounds.

\begin{tabular}{lc}
\hline \hline \multicolumn{1}{c}{ Background } & Number of expected events \\
\hline$Z+\gamma \rightarrow \nu \bar{\nu} \bar{\nu}+\gamma$ & $12.1 \pm 1.3$ \\
$W \rightarrow e \nu$ & $3.8 \pm 0.3$ \\
Noncollision & $2.8 \pm 1.4$ \\
Misidentified jets & $2.2 \pm 1.5$ \\
$W+\gamma$ & $1.5 \pm 0.2$ \\
Total Background & $22.4 \pm 2.5$ \\
Data & 29 \\
\hline \hline
\end{tabular}

Finally, the $e / \gamma$ template is obtained from a data sample of isolated electrons.

The total number of background events from misidentified jets $\left(N_{\text {misid }}\right)$ can be predicted from the fake photon sample based on the rates at which jets, passing all other photon identification criteria, fail or pass the track isolation requirement. To measure those rates we use an EM plus jet sample, where the EM object passes all photon identification requirements except the track isolation, and where the jet approximately balances the EM object in the transverse plane. We first determine the number of events $\left(N_{1}\right)$ in the sample that fail the track isolation requirement. We then fit the DCA distribution of the events that pass the track isolation to a linear sum of the $e / \gamma$ and misidentified jets templates in order to extract the number of misidentified jets $\left(N_{2}\right)$ passing the track isolation. $N_{\text {misid }}$ is then equal to the number of events in the fake photon sample multiplied by $N_{2} / N_{1}$. We fit the DCA distribution in the photon sample to a linear sum of the three templates, fixing the contribution of misidentified jets as described above, and determine the $e / \gamma$ and noncollision contributions. The result of the fit is illustrated in Fig. 1. Most of the signal photons have DCA less than $4 \mathrm{~cm}$, therefore we limit our

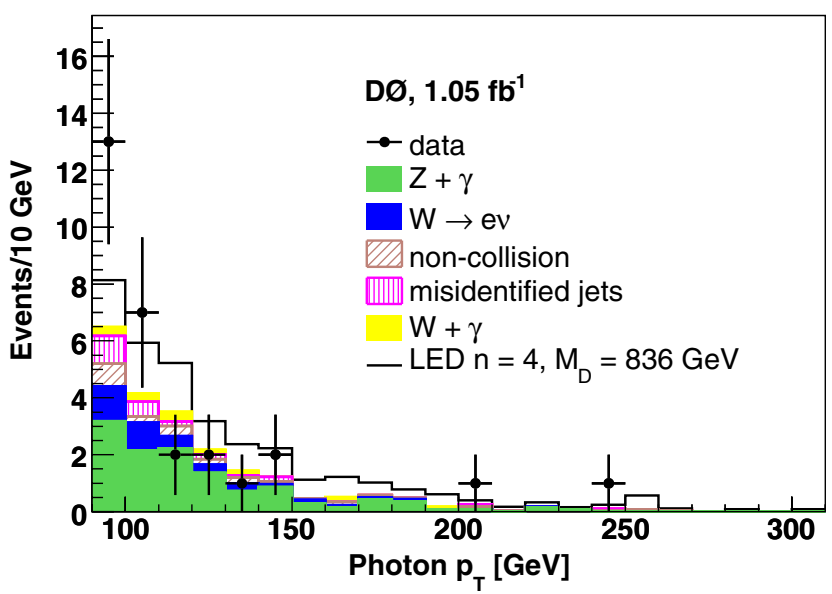

FIG. 2 (color online). Photon $p_{T}$ distribution for the final candidate events (data points show statistical uncertainties), after all the selection requirements. The LED signal is stacked on top of SM backgrounds. 
TABLE II. Summary of limit calculations.

\begin{tabular}{cccc}
\hline \hline$n$ & Signal efficiency & Observed (expected) cross section limit (fb) & Observed (expected) limit (GeV) \\
\hline 2 & $0.49 \pm 0.04$ & $27.6(23.4)$ & $884(921)$ \\
3 & $0.48 \pm 0.04$ & $24.5(22.7)$ & $864(877)$ \\
4 & $0.47 \pm 0.04$ & $25.0(22.8)$ & $836(848)$ \\
5 & $0.43 \pm 0.04$ & $25.0(24.8)$ & $820(821)$ \\
6 & $0.50 \pm 0.05$ & $25.4(22.3)$ & $797(810)$ \\
7 & $0.49 \pm 0.04$ & $24.0(23.1)$ & $797(801)$ \\
8 & $0.52 \pm 0.05$ & $24.2(21.9)$ & $778(786)$ \\
\hline \hline
\end{tabular}

analysis to this particular window, which contains 29 data events.

The only physics background to the $\gamma+\not ̈_{T}$ final state is the process $Z+\gamma \rightarrow \nu \bar{\nu}+\gamma$. This irreducible contribution is estimated from a sample of Monte Carlo (MC) events generated with PYTHIA using CTEQ6L1 parton distribution functions (PDFs) [13]. The main instrumental background arises from $W \rightarrow e \nu$ decays, where the electron, due to tracking inefficiency or hard bremsstrahlung, is misidentified as a photon. This contribution is estimated from data using a sample of isolated electrons. The same requirements as for the photon sample are imposed, and the remaining number of events is scaled by $\left(1-\epsilon_{\text {trk }}\right) / \epsilon_{\text {trk }}$, where $\epsilon_{\text {trk }}$ is the track reconstruction efficiency of $(98.6 \pm$ $0.1) \%$ [14]. A smaller instrumental contribution to the background is expected from $W+\gamma$ production where the charged lepton in a leptonic $W$ boson decay is not detected. The kinematics of this contribution is obtained from $W(+$ jets $) \rightarrow$ lepton $+\nu(+$ jets $)$ MC samples generated with PYTHIA, while the cross section is taken from the MC generator based on [15], which predicts all contributions (initial state radiation, trilinear gauge boson vertex, and final state radiation) to the full process. We generate signal events [16] with $M_{D}=1.5 \mathrm{TeV}$ for $n=2,3,4,5,6$, 7 , and 8. For different values of $M_{D}$, the cross section scales as $1 / M_{D}^{n+2}$, leaving the kinematic spectra unaffected for a fixed number of extra dimensions.

All MC events are passed through a detector simulation based on the GEANT [17] package, and processed using the same reconstruction software as for the data. Additionally, we apply scale factors, with values ranging from $94 \%$ to $98 \%$, to account for the differences between the efficiency determinations from data and simulation.

The main sources of systematic uncertainty are the uncertainty in the photon identification efficiency (5\%), the uncertainty in the total integrated luminosity $(6.1 \%)$, and the uncertainty in the signal acceptance from the PDFs $(4 \%)$.

For the SM backgrounds estimated from MC, the quoted uncertainties include the uncertainty in the theoretical cross section, which is dominated by the uncertainty in the next-to-leading-order $K$ factors (7\%). For the range of $p_{T}$ in question and for the selection requirements used in this analysis, the $K$ factors vary around unity within this uncertainty margin $[15,18]$. The uncertainty in the width of the $e / \gamma$ sample DCA template results in an additional systematic uncertainty of 0.4 events in the noncollision background estimate.

The final numbers of events for data and backgrounds are given in Table I. Figure 2 shows the photon $p_{T}$ distribution, with the SM backgrounds stacked on top of each other. Data and the SM expectation agree, so we proceed to set lower limits for the fundamental Planck scale $M_{D}$. We employ the modified frequentist approach [19] to set limits on the production cross section for the signal. This method is based on a log-likelihood ratio test statistic and uses the binned photon $p_{T}$ distribution. Assuming the leading-order theoretical cross section for the signal, we derive the following lower limits on $M_{D}$ at the 95\% C.L.: $M_{D}>$ $884,864,836,820,797,797$, and $778 \mathrm{GeV}$ for $n=2,3$, 4, 5, 6, 7 and 8 extra dimensions, respectively. Table II and Fig. 3 summarize the results for the limit calculations.

To conclude, we have conducted a search for LED in the $\gamma+\mathbb{E}_{T}$ channel, finding no evidence for their presence. We have set limits on the fundamental Planck scale, significantly improving results of previous searches.

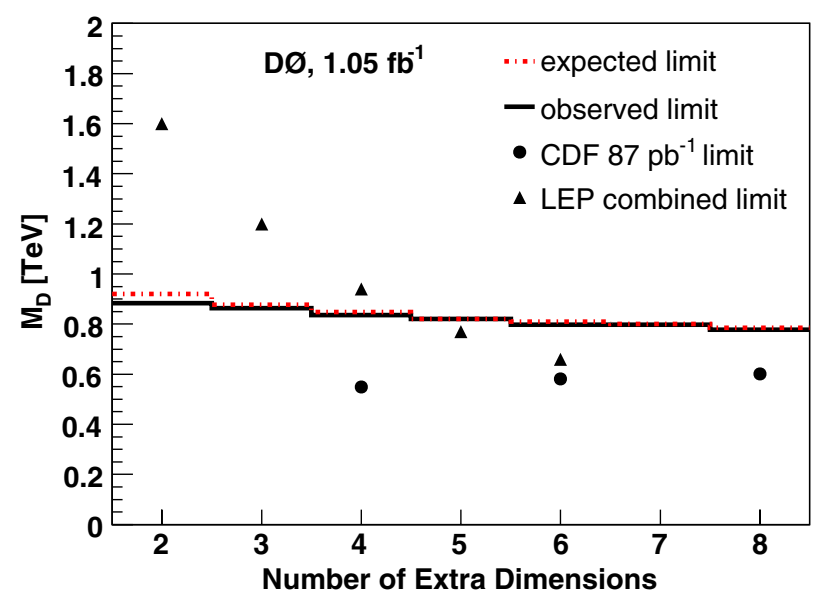

FIG. 3 (color online). Expected and observed lower limits on $M_{D}$ for LED in the $\gamma+\not E_{T}$ final state. CDF limits with $87 \mathrm{pb}^{-1}$ of data [3], and the LEP combined limits [6] are also shown. 
We thank Stephen Mrenna for his help with generating MC signal events, the staffs at Fermilab and collaborating institutions, and acknowledge support from the DOE and NSF (USA); CEA and No. CNRS/IN2P3 (France); FASI, Rosatom and RFBR (Russia); CNPq, FAPERJ, FAPESP and FUNDUNESP (Brazil); DAE and DST (India); Colciencias (Colombia); CONACyT (Mexico); KRF and KOSEF (Korea); CONICET and UBACyT (Argentina); FOM (The Netherlands); STFC (United Kingdom); MSMT and GACR (Czech Republic); CRC Program, CFI, NSERC and WestGrid Project (Canada); BMBF and DFG (Germany); SFI (Ireland); The Swedish Research Council (Sweden); CAS and CNSF (China); and the Alexander von Humboldt Foundation.

*Visitor from Augustana College, Sioux Falls, SD, USA. ${ }^{\dagger}$ Visitor from The University of Liverpool, Liverpool, United Kingdom.

${ }^{\ddagger}$ Visitor from ICN-UNAM, Mexico City, Mexico.

${ }^{\S}$ Visitor from II. Physikalisches Institut, Georg-AugustUniversity, Göttingen, Germany.

"Visitor from Helsinki Institute of Physics, Helsinki, Finland.

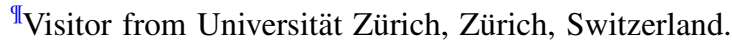

**Deceased.

[1] N. Arkani-Hamed, S. Dimopoulos, and G. Dvali, Phys. Lett. B 429, 263 (1998).

[2] G. Guidice, R. Rattazzi, and J. Wells, Nucl. Phys. B544, 3 (1999).
[3] D. Acosta et al. (CDF Collaboration), Phys. Rev. Lett. 89, 281801 (2002).

[4] B. Abbott et al. (D0 Collaboration), Phys. Rev. Lett. 86, 1156 (2001); 90, 251802 (2003); ibid.95, 161602 (2005).

[5] D. Acosta et al. (CDF Collaboration), Phys. Rev. Lett. 92, 121802 (2004).

[6] LEP Exotica Working Group, URL: http://lepexotica.web. cern.ch/LEPEXOTICA/notes/2004-03/ed_note_final .ps.gz, and references therein.

[7] V. M. Abazov et al. (D0 Collaboration), Nucl. Instrum. Methods Phys. Res., Sect. A 565, 463 (2006).

[8] Pseudorapidity is defined as $-\ln [\tan (\theta / 2)]$, where $\theta$ is the angle between the particle and the proton beam direction.

[9] S. Abachi et al. (D0 Collaboration), Nucl. Instrum. Methods Phys. Res., Sect. A 338, 185 (1994).

[10] T. Andeen et al., Report No. FERMILAB-TM-2365 (2007).

[11] G. C. Blazey et al., arXiv:hep-ex/0005012.

[12] T. Sjöstrand et al., Comput. Phys. Commun. 135, 238 (2001).

[13] J. Pumplin et al., J. High Energy Phys. 07 (2002) 012; D. Stump et al., ibid. 10 (2003) 046.

[14] V. M. Abazov et al. (D0 Collaboration), Phys. Lett. B 659, 856 (2008).

[15] U. Baur and E. L. Berger, Phys. Rev. D 41, 1476 (1990).

[16] Stephen Mrenna (private communication).

[17] R. Brun and F. Carminati, CERN Program Library Long Writeup Report No. W5013, 1994.

[18] U. Baur, T. Han, and J. Ohnemus, Phys. Rev. D 57, 2823 (1998).

[19] W. Fisher, Report No. FERMILAB-TM-2386-E, 2007; T. Junk, Nucl. Instrum. Methods Phys. Res., Sect. A 434, 435 (1999); A. Read, CERN Report No. 2000-005, 2000. 